\title{
Prostate Cancer Biomarkers- A Bench to Bedside Perspective
}

\author{
Mourad Abouelleil, Gennady Bratslavsky and Dimitra Bourboulia* \\ Department of Urology, SUNY Upstate Medical University, USA
}

Received: December 17, 2013; Accepted: January 17, 2014; Published: January 31, 2014

"Corresponding author: Dimitra Bourboulia, Department of Urology and Department of Biochemistry \& Molecular Biology, SUNY Upstate Medical University, 750 East Adams Street, Syracuse, NY 13210, USA, Tel: 315-464-8712; Fax: 315-464-8750; E-mail: bourmpod@upstate.edu

\section{Prostate cancer: current status}

Prostate cancer (PCa) is the most common non-cutaneous malignancy in men and is responsible for the second most common age-adjusted death rate due to cancer among men in the US, after lung cancer [1]. The prevalence of PCa increases with age with the chance of detecting PCa in an 80 year-old male being as high as $60 \%$ [2]. While PCa is generally considered a disease of "old age" with most men dying with and not from PCa, the mortality from PCa is still high, with estimated one PCa death globally every four minutes [3].

Clinically, there is an arsenal of tools for managing or treating PCa, including surgical removal of the prostate, radiation, hormone deprivation, ablative therapies, or active surveillance. Unfortunately, side effects and complications from treatment are not benign and depending on the treatment modality may include urinary incontinence, erectile dysfunction, hemorrhagic cystitis and lower urinary tract symptoms (LUTS). Currently, ProstateSpecific Antigen (PSA) screening and Digital Rectal Exams (DRE) are most commonly used and widely available means of screening, and recommended by the American Urological Association (AUA) [4].

\section{PSA: friend or foe?}

PSA is used as a screening marker of PCa. A recent large multi-institutional randomized trial looking at the relationship between PSA screening and PCa mortality, published in the New England Journal of Medicine (NEJM), demonstrated that PSA-based screening reduced the rate of death from PCa by $20 \%$ but was also associated with a high risk of over diagnosis [5]. In a follow-up analysis, it was shown that PSA screening reduced mortality from PCa but did not affect all-cause mortality in the screened group compared to the controls that were not offered any screening [6]. Moreover, an American randomized, controlled trial was done in 10 centers, randomizing 76,693 men to screening or no screening via DRE and PSA and determined the effects on mortality from PCa during the first 7 to 10 years of the study. The conclusion of this study was that there was no significant difference between the two groups in rate of death from PCa [7]. Given the comprehensive review on the above scientific data, and the fact that PSA is not the perfect marker with less than perfect sensitivity and specificity for diagnosing of PCa, Based on the evidence from several clinical trials and the fact that PSA is not the perfect marker, in terms of sensitivity and specificity for diagnosing PCa, the U.S. Preventive Services Task Force no longer recommends PSA screening for healthy men [8]. Clearly, this recommendation applies to the general male population of the Unites States and by no means includes patients after being diagnosed with or treated of PCa.

\section{Challenges to address in prostate cancer}

\section{Biomarker discovery}

The task at hand is to be able to both diagnose patients with PCa using highly sensitive and specific methods, as well as, identify patients who will receive the best prognosis and benefit from PCa treatment. It is therefore essential, to find patients with a low-grade indolent cancer who will not die from PCa and spare them from unnecessary intervention. Identification of novel biomarkers with a clear understanding of their role in disease development and progression is paramount. Still, there is a plethora of reports trying to address this challenge, and no perfect marker for PCa exists till date. The tumor marker list includes candidate proteins specific to PCa cells, common genetic markers, circulating miRNAs and recently, proteins secreted by the tumor cells that comprise the PCa secretome have been added to the list. A clinically relevant, newly identified, PCa biomarker should be sensitive and specific, preferably secreted in the plasma, urine or semen, to facilitate its detection. The diagnostic assay based on this biomarker needs to be relatively low-cost to become an effective screening tool that is readily available to the public. In this paper, we focus our attention on secreted proteins and cellular products in body fluids (eg. blood, urine), as potentially the most applicable for PCa screen (in diagnosis, prognosis and treatment).

Despite its limitations, the most accepted and frequently used secreted biomarker for PCa is PSA (Table 1). PSA is a serine protease enzyme secreted by the prostate gland to liquefy semen [9]. The protein is very specific to the prostate gland but is not specific to PCa. In patients who underwent prostatectomy for PCa, PSA is an excellent test for recurrence [10]. Most recently, PHI (prostate health index) has been approved by the FDA for clinical evaluation of PCa diagnosis. PHI has also demonstrated, better prediction of the risk of aggressive cancer. It combines 


\begin{tabular}{|l|l|l|l|}
\hline Biomarker & Molecular weight (kD) & Clinical use & Characteristics \\
\hline AGR2 & $18-21$ & $\begin{array}{l}\text { Promising for screening but not used } \\
\text { clinically at this point. }\end{array}$ & $\begin{array}{l}\text { Highly expressed in cancer cells. Plays a role in down } \\
\text { regulation of p53 [14]. }\end{array}$ \\
\hline AMACR & 47 & $\begin{array}{l}\text { Used in screening for men who had } \\
\text { repeated TRUS biopsy. }\end{array}$ & $\begin{array}{l}\text { Mitochondrial enzyme, urine sediment is collected after } \\
\text { DRE looking for the expressed RNA [16]. }\end{array}$ \\
\hline EN2 & 33 & $\begin{array}{l}\text { Screening for BRAC1/2, high risk } \\
\text { patients for PCa development. }\end{array}$ & $\begin{array}{l}\text { Secreted marker in urine. ELISA kit is used for detection } \\
\text { [15]. }\end{array}$ \\
\hline PCA3 & Non-coding RNA & $\begin{array}{l}\text { Secreted, used in men undergoing } \\
\text { repeated prostate biopsy at this point. }\end{array}$ & $\begin{array}{l}\text { Secreted RNA that is over expressed in PCa cells. Urine } \\
\text { collected after a rectal exam and prostatic massage; is } \\
\text { highly specific for PCa [12]. }\end{array}$ \\
\hline PSA & 34 & $\begin{array}{l}\text { Secreted, serum diagnostic test. Used in } \\
\text { screening and recurrence. }\end{array}$ & $\begin{array}{l}\text { Secreted serine protease enzyme; very specific to the } \\
\text { prostate gland, but not specific to PCa [9]. }\end{array}$ \\
\hline TMPRSS: ERG & 49 & $\begin{array}{l}\text { Promising marker but not used clinically } \\
\text { at this point. }\end{array}$ & $\begin{array}{l}\text { Genetic alteration: ERG gene fusion is highly specific to } \\
\text { PCa [17]. }\end{array}$ \\
\hline
\end{tabular}

Table 1: Current and promising prostate cancer biomarkers.

Note: TRUS-Transrectal Ultrasound; DRE-Digital Rectal Exam; kD-Kilodaltons

measurements of total PSA, free PSA and pro-PSA and is estimated to be 2.5 times more specific in detecting PCa than screening for PSA alone [11].

PCA3 (prostate cancer antigen 3) has been approved by the FDA and is currently being used as a biomarker for PCa and in a molecular test to diagnose PCa. It it is a non-coding RNA that is over expressed in PCa and is usually found in urine collected upon post-prostatic massage. It is highly specific for PCa but has low sensitivity [12]. Most recently, PCA3 was compared to PSA in identifying patients with PCa. PCA3 out performed PSA in predicting PCa in low and intermediate grade but not in high grade cancer [13].

Recent studies have reported promising protein markers that may be clinically applicable to PCa in the future. AGR2 (Anterior gradient homolog 2) is associated with many cancers and plays a role in the down regulation of tumor suppressor protein p53. Further investigation is needed to understand its possible use in PCa screening [14]. Moreover, a member of the HOX gene family EN2 (Engrailed-2) was evaluated in the urine of men with genetically high risk mutations BRCA1 and BRCA2. The results were quite promising: high levels of EN2 were associated with PCa diagnosis. The ELISA detection method used demonstrated sensitivity of $66.7 \%$ and specificity of $89.3 \%$ [15]. Interestingly, the data did not show statistically significant association between EN2 levels and carrying BRACA1/2 mutation. AMACR (Alphamethylacyl-CoA Racemase) is a mitochondrial enzyme that is over expressed in PCa. Urine sediment from patients is collected after digital rectal exam (DRE); the assay detects total RNA isolated to determine expression of the AMACR. It can be combined with PCA3 to improve sensitivity and specificity [16]. Finally, a gene fusion such as that seen in genetic alteration TMPRSS2:ERG was found to be $70 \%$ specific to PCa in a hospital based cohort done in 2007 [17]. TMPRSS2: ERG is currently under evaluation for its diagnostic and prognostic values.

\section{Application of PCa biomarkers in the clinic}

Being able to make the diagnosis of PCa is important, however, being able to differentiate between low-grade and high-grade cancer is also invaluable. Currently, Gleason score is the most widely used tool in assigning PCa grade and prostate aggressiveness. However, even low-grade tumors, in some circumstances, while not all high-grade cancers, will become metastatic. The best tool in this case would be a test that can assess the tumor metastatic potential. It can be a surveillant and prognostic tool for patients with PCa to mark disease progression. Table 1 summarizes currently used and promising targets.

Presently, PSA is the only FDA approved, clinically used PCa biomarker detected in serum; however, over the last decade, there have been enormous efforts to discover novel PCa specific tumor markers. The National Cancer Institute (NCI) has provided carefully designed initiatives that promote identification and translation of biomarker discovery to the clinic, for accomplishing this difficult task. Innovative strategies involve integrated approaches in the discovery of novel biomarkers including state of the art proteomic technologies. Modern mass spectrometry (MS) has the potential not only to identify new proteins but also to determine specific interaction partners. Similar to genomic microarray technology, proteomic analysis now comprises the discovery of biomarkers, determination of global interactomes and uses multi-dimensional data analysis tools for data processing. Looking at the clinical application, secretome analysis using upto-date proteomic tools could provide information on biomarker function and signaling pathways involved: knowledge essential in finding not only a biomarker but also targeted therapy [18].

In reality, the future of screening for $\mathrm{PCa}$, either in a diagnostic or prognostic manner, will likely be a panel of biomarkers which increase the sensitivity and specificity of the diagnosis or prognosis over each individual marker's performance. The approach is similar to the panel of biomarkers for testicular cancer diagnosis and treatment guidance. In testicular cancer, for example, the use of LDH, BHCG, and AFP (lactate dehydrogenase, beta human chorionic gonadotropin, alpha fetoprotein) allow for suspicion of specific tumor type and tumor burden [19]. We 
predict that for PCa screening we may have to combine multiple assays to be able to make accurate diagnosis; unless we can find the perfect biomarker that can be both highly sensitive and specific in diagnosing PCa.

\section{References}

1. American Cancer Society (2013) Cancer facts \& figures 2013. Atlanta.

2. Zlotta AR, Egawa S, Pushkar D, Govorov A, Kimura T, et al. (2013) Prevalence of prostate cancer on autopsy: cross-sectional study on unscreened Caucasian and Asian men. J Natl Cancer Inst 105(14): 1050-1058.

3. Siegel R, Naishadham D, Jemal A (2012) Cancer statistics, 2012. CA Cancer J Clin 62(1): 10-29.

4. Carter HB, Albertsen PC, Barry MJ, Etzioni R, Freedland SJ, et al. (2013) Early detection of prostate cancer: AUA Guideline. J Urol 190(2): 419426.

5. Schröder FH, Hugosson J, Roobol MJ, Tammela TL, Ciatto S, et al. (2009) Screening and prostate-cancer mortality in a randomized European study. N Engl J Med 360(13): 1320-1328.

6. Schröder FH, Hugosson J, Roobol MJ, Tammela TL, Ciatto S, et al (2012) Prostate-cancer mortality at 11 years of follow-up. N Engl J Med 366(11): 981-990.

7. Andriole GL, Crawford ED, Grubb RL III, Buys SS, Chia D, et al. (2009) Mortality results from a randomized prostate-cancer screening trial. N Engl J Med, 360(13): 1310-1319.

8. Calonge N, (2012) U.S. Preventive services task force U.S. preventive services task force recommendation statement screening for prostate cancer.

9. Balk SP, Ko YJ, Bubley GJ (2003) Biology of prostate-specific antigen. J Clin Oncol 21(2): 383-391.

10. Abramowitz MC, Li T, Buyyounouski MK, Ross E, Uzzo RG, et al. (2008).
The Phoenix definition of biochemical failure predicts for overall survival in patients with prostate cancer. Cancer 112(1): 55-60.

11. Nichol MB, Wu J, Huang J, Denham D, Frencher SK, et al. (2012) Costeffectiveness of Prostate Health Index for prostate cancer detection. BJU Int 110(3): 353-362.

12. Haese A, de la Taille A, van Poppel H, Marberger M, Stenzl A, et al. (2008). Clinical utility of the PCA3 urine assay in European men scheduled for repeat biopsy. Eur Urol 54(5), 1081-1088.

13. Chevli KK, Duff M, Walter P, Yu C, Capuder B, et al. (2013). Urinary PCA3 as a predictor for prostate cancer in a cohort of 3073 men undergoing initial prostate biopsy. J Urol S0022-5347(13): 06087-4.

14. Qian WJ, Shi T, Gao Y, Quek SI, Fillmore TL, et al. (2013). A highly sensitive targeted mass spectrometric assay for quantification of AGR2 protein in human urine and serum. J Proteome Res doi: $10.1021 /$ pr400912c.

15. Killick E, Morgan R, Launchbury F, Bancroft E, Page E, et al. (2013). Role of Engrailed-2 (EN2) as a prostate cancer detection biomarker in genetically high risk men. Sci Rep 3: 2059.

16. Ouyang B, Bracken B, Burke B, Chung E, Liang J, et al. (2009) A duplex quantitative polymerase chain reaction assay based on quantification of alpha-methylacyl-CoA racemase transcripts and prostate cancer antigen 3 in urine sediments improved diagnostic accuracy for prostate cancer. J Urol 181(6): 2508-2513.

17. Mehra R, Tomlins SA, Shen R, Nadeem O, Wang L, et al. (2007) Comprehensive assessment of TMPRSS2 and ETS family gene aberrations in clinically localized prostate cancer. Mod Pathol 20(5), 538-544.

18. Ahmad Y, Lamond AI (2013) A perspective on proteomics in cell biology. Trends Cell Biol S0962-8924(13): 00191-8.

19. Ruf CG, Sachs S, Khalili-Harbi N, Isbarn H, Wagner W, et al. (2013) Prediction of metastatic status in non-seminomatous testicular cancer. World J Urol doi: 10.1007/s00345-013-1194-0. 